\title{
Competition Over Timeline in Social Networks
}

\author{
Eitan Altman \\ Parmod Kumar \\ INRIA Sophia-Antipolis, 2004 Route des \\ Lucioles, 06902 Sophia-Antipolis Cedex, France \\ eitan.altman@inria.fr \\ parmod.kumar@inria.fr
}

\author{
Srinivasan Venkatramanan \\ Anurag Kumar \\ Indian Institute of Science \\ Bangalore, India \\ vsrini@ece.iisc.ernet.in \\ anurag@ece.iisc.ernet.in
}

\begin{abstract}
Social networking sites pervade the World Wide Web and have millions of users worldwide. This provides ample opportunity for brands and organisations to reach out to a large and diverse audience. They do so by creating content and spreading it across the social network. Most popular social networks follow a timeline based homepage to display such content to the end users. Content once posted on the timeline, remains visible for a limited time, determined by the rate of content generation in the network. There are various ways by which brands can become more visible on the timeline of their followers, for instance by retransmitting/advertising their content from time to time. Hence, with multiple content creators in the network, there is a competition over a user's timeline, which we analyse in this paper. We first characterise the occupancy distribution of a given user's timeline and then use queueing techniques to analyse the period of time a content is present on a given timeline. We then study the competition between different content creators and characterise the equilibrium rate of content generation. We finally provide some numerical results, which provide insights into the effect of various system parameters.
\end{abstract}

\section{INTRODUCTION}

Social media encompasses a wide range of online tools that enable and enhance social interactions among its users. Some examples include email, chat, discussion forums, blogs, social networking sites and multiplayer gaming communities. Using these online tools, users can work together and create, find, share, evaluate, and make use/sense of the large amount of information available online in varied forms. A brief description of some popular types of social media, their structure and function, can be found in [1].

Most online social networks are based on real world relationships such as friendship (Facebook), professional contacts (LinkedIn), etc. Recently, networks like Twitter and Google+ have extended beyond offline relationships and are perceived as content-centric social networks. In general, an online social network allows users to create their profiles, make connections with other users, share and discover new content, etc. [1]. Over the past decade, the number of account holders in online social networks (OSNs) has increased by orders of magnitude [2]. With the huge success of major OSNs like Facebook ( $>1$ billion users), Google+ (343 million users) and Twitter (300 million users), social networking has become an integral part of the Internet [3]. With the advent of low-cost smartphones, and mobile apps available for social networking, user engagement on these sites is bound to increase. Hence, it comes as no surprise that, most brands and organisations have migrated to social media from other traditional media for their advertising campaigns. This leads to competition for user attention on the social networks. Due to the availability of data, several empirical studies have been carried out in the past by organizations, but the analysis of competition dynamics on these social networks have not been given sufficient attention in the literature.

An interesting feature to observe is that, all three major online social networks (Facebook, Google+, Twitter) use a timeline based template in their homepages (see Figure (1)). In Facebook, this is referred to as News Feed, while in Twitter it is referred to as Timeline. In this paper, we will use the term "Timeline" throughout. Though there are several forms of advertising on sites like Facebook, the one that captures most of the user attention is the main timeline. Though very similar to the traditional e-mail inbox view, the ability to place the content (multimedia/hyperlinks) directly on the timeline provides a more engaging experience for the end user. Since users are connected (subscribe/follow/friend) to other users and brands' accounts on these sites, content pushed out by these accounts reach the user timeline. Facebook and Google+ employ algorithms to sort the timeline and prioritise feeds (according to the learned preferences from past user interactions), while Twitter still provides a reverse chronological view. In this work, we shall assume a reverse chronological timeline.

Though technically a user can scroll through a very large number of posts on his/her timeline, it is safe to assume that for practical purposes that the timeline has a fixed size. Recent research using eye-tracking technology, and studies on user scrolling behavior have confirmed the fact that user attention is focused mostly on the first few entries. In summary, Web users spend $80 \%$ of their time looking at information above the page fold. Although users do scroll, they allocate only $20 \%$ of their attention below the fold [4] (see Figure (2)). Hence, in this work, we restrict ourselves to fixed timeline size. It would be an interesting future direction to treat the timeline size for each user as a random variable, arising out of a known distribution.

Thus a message received on the timeline of a user is only visible for a limited period of time, until it is pushed down by posts arriving later. Content creators would want their posts to stay longer on the timeline, and as a result take 


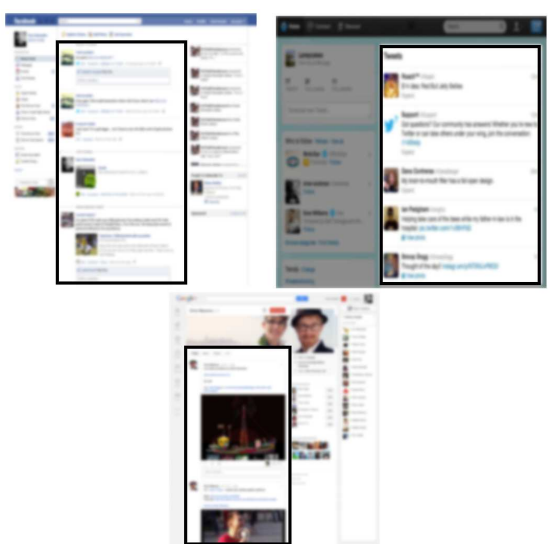

Fig. 1. Homepage snapshots from Facebook, Twitter, Google+. The timelines are highlighted in bold black lines. The images have been blurred to protect private $\mathrm{d}^{\mathrm{n}+\mathrm{r}}$

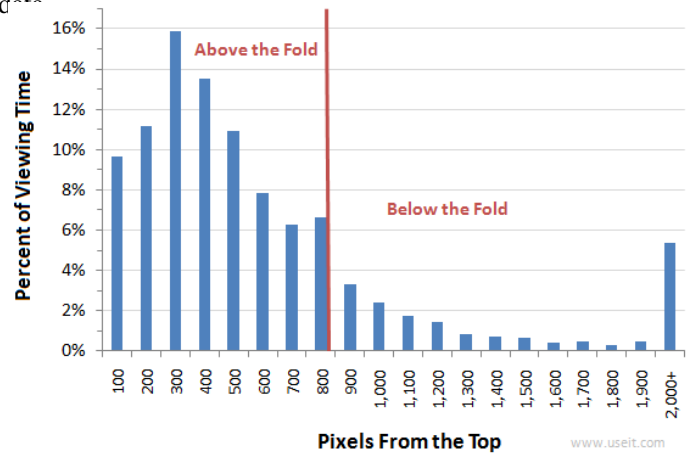

Fig. 2. Distribution of user fixations on webpages (not restricted to social networks) along stripes that were 100 pixels tall. This justifies our assumption of considering a fixed timeline size. (Figure reproduced from [4])

actions such as resending the same message, advertising the post sent, etc. This returns the content to the top of the timeline. Also, when users view a particular content on their timeline, they have the option to forward it to their friends ("Share" on Facebook, "Retweet" on Twitter), thus making it visible on their timelines. Therefore, there is a competition for limited timeline space by several content creators. Another interesting notion is that the social network providers (i.e., Facebook, Google+, Twitter, etc.) have complete control over the parameters like the size of the timeline, and can set policies that roughly control the topology of the network. Hence it would also be interesting to study, if they can choose the right parameters to maximise their revenue (paid by the content creators for advertising their content).

Related work: Past work in this area have focused on the competition between contents in social networks over popularity and over visibility space, in conjunction with advertisement issues to promote content. Both fully dynamic [5] models as well as semi-dynamic [6] models have been proposed. It has been noted that these problems are similar in nature to the problem of competition over shelf space, a problem well studied in Operations Research (see [7]). A fluid deterministic game approach has been proposed in [8] to approximate the competition over followers' timeline when the number of players become large. In the following section, we provide a brief overview of some terminologies associated with Facebook, currently the most popular social networking site.

Facebook Terminologies [9]

Timeline: In Facebook, the name "Timeline" has a special meaning, and refers to a user's personal profile, containing information such as the user's education/employment history, relationship status, his recent posts and shared content.

Friend: One can add other users as his/her friends on mutual consent. The links thus established are undirected.

Subscribe: Unlike friends, this feature enables users to follow pages/accounts that are held by celebrities or brands, thus receiving regular updates. Most brands use Facebook to engage with, share deals and seek feedback from their consumers. Any user can make this option active on his/her profile and a subscriber to that profile will receive all public updates from the user.

News Feed: In this work, when we refer to timeline, we are actually referring to the News Feed in Facebook. All contents published by a user's friends and subscribed pages appear on the News Feed. This also appears as the homepage after login.

The Status Update: This allows users to post content to their friends and followers. This can contain text, images, videos or hyperlinks, and appear on the News Feed of his/her friends.

Like/Comment/Share: Almost all the types of Facebook content have these three buttons. While "liking" a content shows support, "commenting" allows interaction with the content creator and "sharing" forwards the content to a user's friends and followers. A recent update on Facebook shows "Like" and "Comment" activities on a Ticker tape (on the top right of the homepage) while "Share" activities show up in the main News Feed.

In the next section we present the social network model and the model for the timeline of a particular user on Facebook. We then analyse the occupancy distribution of the timeline positions and characterise the expectation duration of $j$ 's content on the timeline of user $i$. In Section IV, we formulate a non-cooperative game among the content creators for timeline space of a single user, and study the equilibrium for both the complete and the partial control case. We finally present numerical results and some insights into the dynamics of competition over the timeline.

\section{TIMELINE MODEL}

Though practically every user in the social network can create and share content, we will restrict our attention in this paper to the setting where we have clear delineation between consumers and content creators (brands and organizations). In the model discussed in this paper, we also assume there is no sharing among users, which will be incorporated in a future extension of the work. Thus, we characterise the content creator-consumer interaction as a bipartite graph, with $\mathcal{I}$ the set of consumers and $\mathcal{J}$ the set of creators. The set of followers (or subscribers) of creator $j$ is denoted by $M_{j} \in \mathcal{I}$. Each consumer in the social network, say $i$, has a set $N_{i} \in \mathcal{J}$ of 


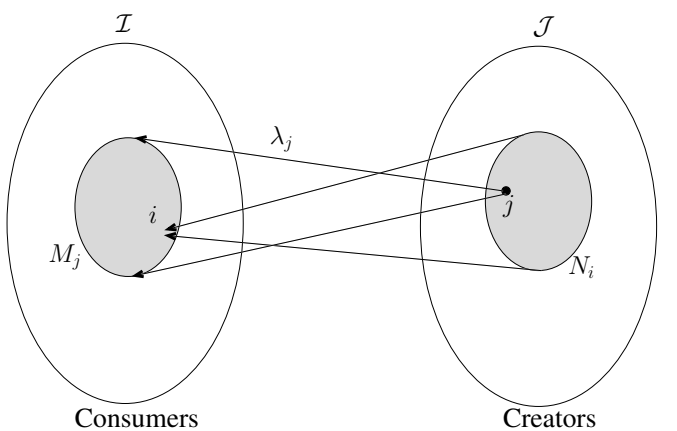

Fig. 3. System model.

sources that it follows (see Figure (3)). In this work, we will be considering only a single user's timeline, and hence in this work, $N_{i}=\mathcal{J}$. Content from source $j$ is created according to a Poisson process with rate/intensity parameter $\lambda_{j}$ and sent to the timelines of all members in $M_{j}$. Define the total rate of content creation in the social network which will appear on the timeline of user $i$ as

$$
\Lambda^{i}=\sum_{j \in N_{i}} \lambda_{j}
$$

Define further

$$
\Lambda_{[-j]}^{i}=\sum_{k \in N_{i}, k \neq j} \lambda_{k}
$$

We are interested in the stationary probability $\pi(i, j)$ of finding a content of source $j$ on the timeline of a user $i, i \in M_{j}$. Assume that at the beginning, i.e., at time 0, there is an arrival of some content $C$ from $j$ to the set of followers $M_{j}$ of user $j$ and therefore to $i$. Assume the timeline of user $i$ has a constant size of $2 K(i)$. Each future arrival to $i$ from $N_{i}$, will push the content $C$ further down, and after $K(i)$ such shifts the content will no longer be visible on the timeline of user $i$. In the meantime, if another post arrives from $j$ to $i$ when $C$ is pushed away from the timeline, there is still a presence of content from $j$ on the timeline of user $i$. We call an $i j$ busy period the time period that starts when a content from $j$ first arrives to the timeline of user $i$ until the instant at which there is no more content from $j$ on the timeline of user $i$. We denote this by $\hat{T}_{i j}$. Before characterising the $i j$-busy period, we introduce the following notation. When $C$ arrives, it is said to be on the top of the timeline, i.e., at position $K(i)$, and at each new arrival, its position gets decremented/shifted by 1 . Let $T_{i j}(k)$ be the time from the instant when $C$ (the content from $j$ on the timeline of $i$ ) enters position $k$ on the timeline until the instant when the timeline of user $i$ does not have any content from $j$. Then $\hat{T}_{i j}=T_{i j}(K(i))$.

\section{ANALYSIS OF OCCUPANCY OF THE TIMELINE}

\section{A. Occupancy distribution}

In this section we obtain the occupancy distribution of the timeline positions. In order to do that, we 2 represent the timeline as a continuous time Markov chain (CTMC) $\mathbf{C}(t)$ taking values in $\mathcal{C}:=\mathcal{J}^{K(i)} . \mathbf{C}(t)$ denotes the vector of contents that occupy the $K(i)$ locations in the timeline of $i$ at time $t$. Then we can state the following theorem.

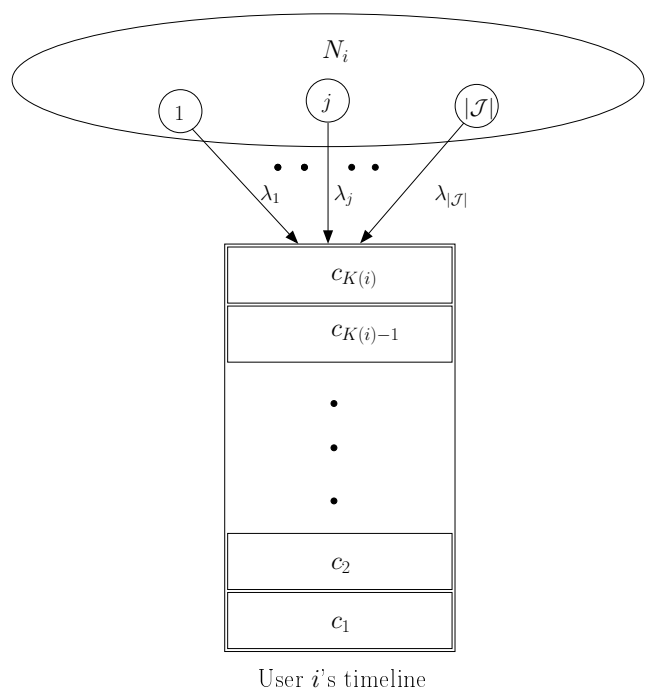

Fig. 4. Schematic of the model for the timeline of user $i$.

Theorem 1. The stationary probability distribution for the CTMC $\mathbf{C}(t)$ is given by,

$$
\pi_{\mathbf{c}}=\Pi_{k=1}^{K(i)} \nu_{c_{k}}
$$

where $\nu_{j}:=\frac{\lambda_{j}}{\Lambda^{i}}$ and $c_{k}$ is the content at the $k$ th position on the timeline.

Proof: Let $Q$ denote the transition rate matrix for the CTMC $\mathbf{C}(t)$. We wish to show that the proposed $\pi_{\mathbf{c}}$ satisfies $\forall \mathbf{c}^{\prime} \in \mathcal{C}$

$$
\sum_{\mathbf{c} \in \mathcal{C}} \pi_{\mathbf{c}} q_{\mathbf{c}, \mathbf{c}^{\prime}}=0
$$

or equivalently,

$$
\pi_{\mathbf{c}^{\prime}} a_{\mathbf{c}^{\prime}}=\sum_{\substack{\mathbf{c} \in \mathcal{C} \\ \mathbf{c} \neq \mathbf{c}^{\prime}}} \pi_{\mathbf{c}} q_{\mathbf{c}, \mathbf{c}^{\prime}}
$$

where $a_{\mathbf{c}}$ is the rate of exiting state $\mathbf{c}$.

The argument that follows holds for the case where not all the elements of $\mathbf{c}^{\prime}$ are the same.

Observe from Figure (4) that there is a possible transition from state $\mathbf{c}$ to $\mathbf{c}^{\prime}$ if and only if the first $(K(i)-1)$ entries of c coincide with the last $(K(i)-1)$ entries of $\mathbf{c}^{\prime}$. If a given $\mathbf{c}^{\prime}$ satifies this condition then we say $\mathbf{c}^{\prime} \in s(\mathbf{c})$. Let $l:=c_{K(i)}^{\prime}$, then the rate $q_{\mathbf{c}, \mathbf{c}^{\prime}}=\lambda_{l}$ if $\mathbf{c}^{\prime} \in s(\mathbf{c})$ and 0 otherwise. The right hand side of Equation (2) then becomes

$$
\begin{aligned}
\sum_{\mathbf{c} \in \mathbf{C}, \mathbf{c} \neq \mathbf{c}^{\prime}} \pi_{\mathbf{c}} q_{\mathbf{c}, \mathbf{c}^{\prime}} & =\sum_{\substack{\mathbf{c} \in \mathcal{C}, \mathbf{c} \neq \mathbf{c}^{\prime} \\
\mathbf{c}^{\prime} \in s(\mathbf{c})}}\left(\Pi_{k=1}^{K(i)} \nu_{c_{k}}\right) \lambda_{l} \\
& =\lambda_{l}\left[\nu_{1}+\cdots+\nu_{|\mathcal{J}|}\right]\left(\Pi_{k=1}^{K(i)-1} \nu_{c_{k}}\right) \\
& =\nu_{l} \Lambda^{i} \Pi_{k=1}^{K(i)-1} \nu_{\mathbf{c}_{k}} \\
& =\pi_{\mathbf{c}^{\prime}} a_{\mathbf{c}^{\prime}}
\end{aligned}
$$

The first equality arises since there are exactly $|\mathcal{J}|$ possible terms in the summation, and subsequently we use the fact that $\nu_{l}=\frac{\lambda_{l}}{\Lambda^{i}}$ and $\sum_{l=1}^{|\mathcal{J}|} \nu_{l}=1$. 
If all the elements of $\mathbf{c}^{\prime}$ are the same, say $\ell$, then the following happens:

$$
\begin{aligned}
& \pi_{\mathbf{c}^{\prime}} a_{\mathbf{c}^{\prime}} \stackrel{?}{=} \sum_{\substack{\mathbf{c} \in \mathcal{C} \\
\mathbf{c} \neq \mathbf{c}^{\prime}}} \pi_{\mathbf{c}} q_{\mathbf{c}, \mathbf{c}^{\prime}} \\
& \nu_{l}^{K(i)} \sum_{j \neq l} \lambda_{j} \stackrel{?}{=}\left(\sum_{\substack{j=1 \\
j \neq l}} \nu_{l}^{(K(i)-1)} \nu_{j}\right) \lambda_{l}
\end{aligned}
$$

which is true since $\nu_{l}=\frac{\lambda_{l}}{\Lambda^{i}}$. In the above equation, there are only $(|\mathcal{J}|-1)$ entries on the right hand side, since $\mathbf{c} \neq \mathbf{c}^{\prime}$, i.e., the last position in cannot be $l$.

\section{B. Expected $i j$-busy period}

In this section, we characterise the expected $i j$-busy period by using a recursion argument. It is also to be noted that, the occupancy distribution derived in the preceding section can also be used to arrive at the same result.

Theorem 2. The expected ij-busy period, the duration for which content from creator $j$ stays on user i's timeline of size $K(i)$, is given by

$$
2 E\left[T_{i j}(K(i))\right]=\frac{1}{\Lambda_{[-j]}^{i}}\left(\frac{1-\alpha^{-K(i)}}{1-\alpha^{-1}}\right)
$$

where

$$
\alpha=\frac{\Lambda_{[-j]}^{i}}{\Lambda^{i}} .
$$

Proof: We have the following recursion for $E\left[T_{i j}(k)\right]$ :

$$
\begin{gathered}
E\left[T_{i j}(0)\right]=0 \\
E\left[T_{i j}(k+1)\right]=\frac{1}{\Lambda^{i}}+\frac{\lambda_{j}}{\Lambda^{i}} E\left[T_{i j}(K(i))\right]+\frac{\Lambda_{[-j]}^{i}}{\Lambda^{i}} E\left[T_{i j}(k)\right]
\end{gathered}
$$

By taking $k=(K(i)-1)$ we obtain after some algebra,

$$
E\left[T_{i j}(K(i))\right]-E\left[T_{i j}(K(i)-1)\right]=\frac{1}{\Lambda_{[-j]}^{i}}
$$

To solve the recursion, let us introduce for $k=1, \ldots, K(i)$

$$
S_{i j}(k):=E\left[T_{i j}(k)\right]-E\left[T_{i j}(k-1)\right]
$$

This satisfies the simpler recursion

$$
S_{i j}(k+1)=\alpha S_{i j}(k)
$$

Hence,

$$
S_{i j}(k+1)=\alpha^{k} S_{i j}(1) .
$$

From Equation (4) we have the condition,

$$
S_{i j}(K(i))=\frac{1}{\Lambda_{[-j]}^{i}} .
$$

Combining last two equations, we get

$$
S_{i j}(1)=\frac{1}{\Lambda_{[-j]}^{i}} \alpha^{-K(i)+1} .
$$

We combine this with the relation:

$$
E\left[T_{i j}(k+1)\right]=S_{i j}(1) \sum_{n=0}^{k} \alpha^{n}
$$

and thus we get,

$$
\begin{aligned}
E\left[T_{i j}(K(i))\right] & =\frac{1}{\Lambda_{[-j]}^{i}} \sum_{n=0}^{K(i)-1} \alpha^{-n} \\
& =\frac{1}{\Lambda_{[-j]}^{i}}\left(\frac{1-\alpha^{-K(i)}}{1-\alpha^{-1}}\right) \cdot 2
\end{aligned}
$$

Note that the expected time during which there is no content from $j$ on the timeline of user $i$ is simply $1 / \lambda_{j}$. Hence the fraction of time that there is content from $j$ on the timeline of user $i$ is given as

$$
\begin{aligned}
p_{i j} & =\frac{E\left[T_{i j}(K(i))\right]}{E\left[T_{i j}(K(i))\right]+1 / \lambda_{j}} \\
& =1-\frac{1}{\lambda_{j} E\left[T_{i j}(K(i))\right]+1} \\
& =1-\frac{1}{q_{j} \sum_{n=0}^{K(i)-1}\left(1+q_{j}\right)^{n}+1} \\
& =1-\frac{1}{\left(1+q_{j}\right)^{K(i)}}
\end{aligned}
$$

where $q_{j}:=\frac{\lambda_{j}}{\Lambda_{[-j]}^{i}}$.

Note that by definition, $1+q_{j}=1 / \alpha$, and thus we have

$$
p_{i j}=1-\alpha^{K(i)}
$$

Note that for $K(i)=1, p_{i j}=\lambda_{j} / \Lambda$.

\section{Social Network TIMEline GAme: COMPUTING THE BEST RESPONSE}

The fraction of time during which content from creator $j$ is present on the timeline of user $i$, is shown in Equation (7), which depends on the rate/intensity parameters $\lambda_{j}, j \in N_{i}$, with which the content is created. Therefore, we assume that for each content creator $j$, the $\lambda_{j}$ are decision variables, satisfying $\lambda_{j} \geq \phi_{j}$, where $\phi_{j}$ is the minimum value of the rate of the content created by source $j$. Player $j$ can put some effort in order to increase the basic arrival rate $\phi_{j}$ of its content to $\lambda_{j}$ at some cost. This together with the rates of the competitors of $j$ will determine the duration of presence of content from $j$ on $i$ 's timeline. We shall seek for the equilibrium values of the variables $\lambda_{j}, j \in N_{i} . \lambda_{j}$ at equilibrium is characterised by the fact that it maximises $W_{j}^{i}$ over $\lambda_{j} \geq \phi_{j}$ where

$$
2 W_{j}^{i}:=p_{i j}-\gamma_{i}\left(\lambda_{j}-\phi_{j}\right)
$$

The Lagrange relaxation gives the minimization of the Lagrangian, substituting for $p_{i j}$ from Equation (7) gives us

$$
L_{j}^{i}=1-\alpha^{K(i)}-\gamma_{j}\left(\lambda_{j}-\phi_{j}\right)+\beta_{j}\left(\lambda_{j}-\phi_{j}\right),
$$


where the Lagrange multipliers $\beta_{j}$ are non-negative. For the best response, we solve

$$
\frac{\partial L_{j}^{i}}{\partial \lambda_{j}}=-K(i) \alpha^{(K(i)-1)}\left(-\frac{\Lambda_{[-j]}^{i}}{\left(\Lambda^{i}\right)^{2}}\right)-\left(\gamma_{j}-\beta_{j}\right)=0
$$

This yields:

$$
\frac{K(i)}{\Lambda^{i}}\left(1-\frac{\lambda_{j}}{\Lambda^{i}}\right)^{K(i)}-\left(\gamma_{j}-\beta_{j}\right)=0
$$

Assume that the minimum is not attained at $\lambda_{j}=\phi_{j}$, then by the complementary slackness conditions, $\beta_{j}=0$.

Thus, the solution of the best response problem is given as

$$
\lambda_{j}=\Lambda^{i}\left[1-\left(\frac{\gamma_{j} \Lambda^{i}}{K(i)}\right)^{\frac{1}{K(i)}}\right]
$$

if it is larger than and equal to $\phi_{j}$, otherwise $\lambda_{j}=\phi_{j}$.

\section{Characterising the EQUilibrium}

Consider the game in which the set of sources $N_{i}$ compete over the timeline of user $i$. Each source is a player and we aim to obtain the Nash equilibrium.

\section{A. Symmetric game}

Consider the symmetric game where the cost parameters are the same for all players, i.e., $\gamma_{j}=\gamma$. Then we have the following theorem.

Theorem 3. At equilibrium each player sends either

$$
\lambda_{j}=\frac{K(i)}{\gamma} \frac{\left(\left|N_{i}\right|-1\right)^{K(i)}}{\left|N_{i}\right|^{(K(i)+1)}}
$$

or $\phi$, whichever is larger. 2

Proof: The condition of symmetry gives us $\Lambda^{i}=\left|N_{i}\right| \lambda_{j}$. Thus

$$
\lambda_{j}=\left|N_{i}\right| \lambda_{j}\left[1-\left(\frac{\gamma\left|N_{i}\right| \lambda_{j}}{K(i)}\right)^{\frac{1}{K(i)}}\right]
$$

which leads to Equation (12).

Also observe that at equilibrium by symmetry, we have $\alpha=$ $\frac{\left|N_{i}\right|-1}{\left|N_{i}\right|}$, thus $\forall j$,

$$
p_{i j}^{*}=1-\left(1-\frac{1}{\left|N_{i}\right|}\right)^{K(i)}
$$

\section{B. Partial control}

In the case of partial control, we assume that only a subset of creators $N_{i}(1) \subset N_{i}$ try to optimise their respective rates while the remaining $N_{i}(2)=N_{i} \backslash N_{i}(1)$ send content at the default minimum rate of $\phi_{2}$. The minimum rate for $j \in N_{i}(1)$ is $\phi_{1}$. The objective is to find the equilibrium rates $\lambda_{j}$ for $j \in N_{i}(1)$. We will then have,

$$
\Lambda^{i}=\sum_{j \in N_{i}(1)} \lambda_{j}+\left|N_{i}(2)\right| \phi
$$

and for $j \in N_{i}(1)$,

$$
\Lambda_{[-j]}^{i}=\sum_{k \in N_{i}(1), k \neq j} \lambda_{k}+\left|N_{i}(2)\right| \phi_{2} .
$$

Proceeding similar to the previous case, we have the best response function $\lambda_{j}$ for the player who participate in the partial control, identical to Equation (11)

$$
2 \lambda_{j}=\Lambda^{i}\left[1-\left(\frac{\gamma_{j} \Lambda^{i}}{K(i)}\right)^{\frac{1}{K(i)}}\right]
$$

When we consider a symmetric game, i.e. $\gamma_{j}=\gamma, \forall j \in$ $N_{i}(1)$, by symmetry argument we have

$$
\Lambda^{i}=\left|N_{i}(1)\right| \lambda+\left|N_{i}(2)\right| \phi_{2}
$$

Thus $\forall j \in N_{i}(1)$,

$$
\begin{aligned}
\lambda_{j}= & \left(\left|N_{i}(1)\right| \lambda_{j}+\left|N_{i}(2)\right| \phi_{2}\right) \cdot \\
& {\left[1-\left(\frac{\gamma\left(\left|N_{i}(1)\right| \lambda_{j}+\left|N_{i}(2)\right| \phi_{2}\right)}{K(i)}\right)^{\frac{1}{K(i)}}\right] }
\end{aligned}
$$

We do not have a closed form solution, and must compute the fixed point numerically. The players play $\lambda_{j}$ or $\phi_{1}$ whichever is larger.

\section{NUMERICAL EXAMPLES}

\section{A. Symmetric complete game}

In this section we study the effect of various system parameters on the equilibrium rate $\lambda$. We will consider the timeline size $K(i)$, number of players $N_{i}$ and the cost parameter $\gamma$. Note that $\lambda$ is a measure of how aggressive the players are, and in real world, the rate of sharing content on the social networks can be measured in units like posts/hour, posts/day, etc. which will depend on the duration for which the user is active on the social network.

The tradeoff parameter $\gamma$ is indicative of the cost paid by the competitors to operate at higher rates than the system minimum $\phi$ (we set $\phi=0$ for the simulations). With respect to the tradeoff parameter, it is straightforward from the expression that $\lambda \propto \frac{1}{\gamma}$, which implies as the cost per unit rate increases, the nodes become less aggressive.

The variations with respect to $K(i)$ and $N_{i}$ show interesting behavior. Figure (5) shows the effect of number of players $N_{i}$ at the equilibrium rate $\lambda$. We observe that for fixed $K(i)$, the players are initially less aggressive for small $N_{i}$ (less competition) and accelerate their aggression as $N_{i}$ increases to a critical value. Beyond the critical $N_{i}$, players become less aggressive since at large $N_{i}$, there are too many competitors for the timeline, that the payoffs $\left(p_{i j}\right)$ become considerably smaller. The acceleration and deceleration of aggressive behavior on either side of the critical $N_{i}$ are more pronounced for smaller $K(i)$.

Figure (6) shows the effect of timeline size $K(i)$ at the equilibrium rate $\lambda$. For fixed $N_{i}$, we see that the players are less aggressive for smaller $K_{i}$ since there are fewer slots on the 


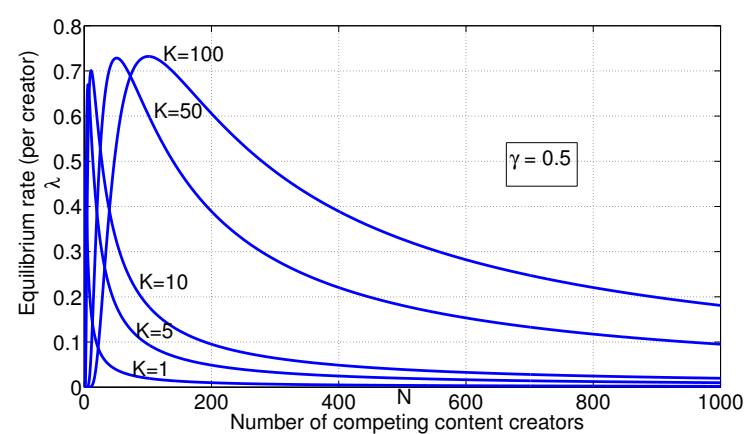

Fig. 5. Variation of equilibrium rate $\lambda$ against $N_{i}$ for $K(i)=$ $1,5,10,50,100$.

timeline to compete for and the payoffs are lesser compared to the cost. As the number of slots on the timeline increase, the players become more aggressive until a critical $K(i)$. Beyond the critical $K(i)$, since there are enough slots on the timeline (for fixed number of players $N_{i}$ ), players become less aggressive. As earlier, the acceleration and deceleration of aggressive behavior on either side of the critical $K(i)$ are more pronounced for smaller $N_{i}$.

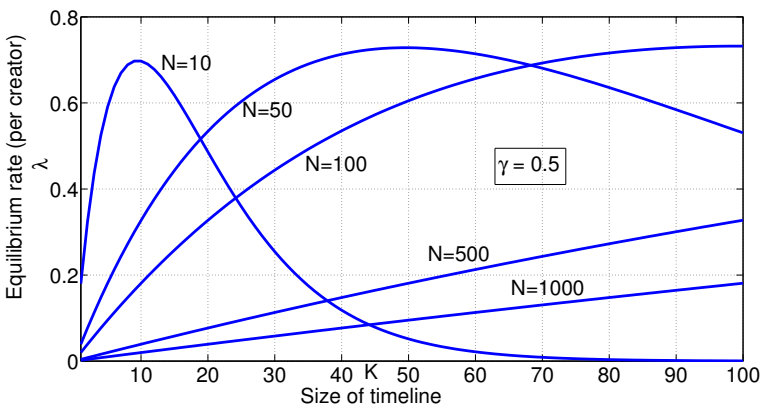

Fig. 6. Variation of equilibrium rate $\lambda$ against $K(i)$ for $N_{i}=$ 10, 50, 100, 500, 1000 .

Observe from the plots that there are critical values of $N_{i}$ and $K(i)$ for which the players' aggression is maximum. Hence, if a social planner has control over $N_{i}$ (by dictating the number of players in the system) and $K(i)$ (by varying the number of posts visible to a consumer in a session), then the social planner can also aim to maximise the total revenue (which is $N_{i} \gamma(\lambda-\phi)$ ). We aim to study this phenomenon in greater detail in our future work.

\section{B. Partial Control}

Since we do not have closed form expression for the equilibrium rates in the partial control case, we will numerically evaluate the fixed point and compare it with the complete game scenario. We study the effect $\frac{N_{i}(1)}{N_{i}}$ (the fraction of participating players) at the equilibrium rate. We set $\phi_{1}=0$, and $\phi_{2}=0.1$, and study the system for $N_{i}=200, K(i)=$ $50, \gamma=0.5$. We observe from Figure (7) that the increase in equilibrium rate with increasing $\frac{N_{i}(1)}{N_{i}}$ is linear and approaches the $\lambda$ of the complete game solution as the fraction of participating players approach 1 .

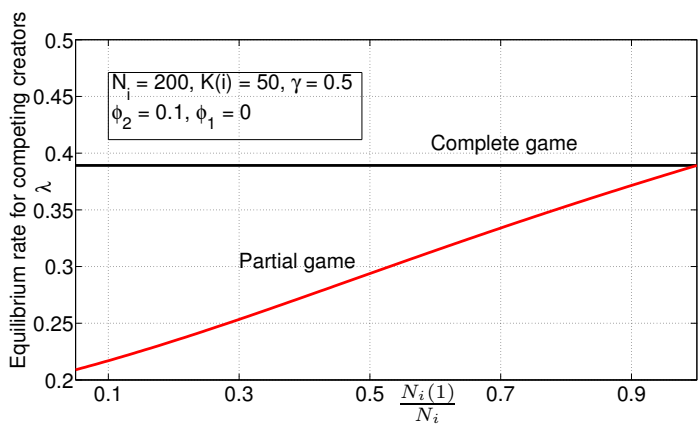

Fig. 7. Variation of equilibrium rate $\lambda$ for the partial control game setting for various values of $\frac{N_{i}(1)}{N_{i}}$, the fraction of competing players.

\section{CONCLUSION}

In this work, we have proposed a tractable model to study the competition dynamics that arise in online social networks, between content creators on a user's timeline. The work can be taken forward in several directions. Although we describe the content on the timelines as Markov chains, we do not formulate the problem as a Markov game but reduce it to a standard convex game. We do not assume knowledge of the state and hence the game becomes simply that of determining the rates $\lambda_{j}$ by the players. In our future work, we also intend to study timelines with variable size, effect of sharing among users and the role of a social planner (the social network provider).

\section{ACKNOWLEDGMENTS}

The authors would like to thank the Department of Science and Technology, India (DST) and the Indo-French Center for the Promotion of Advanced Research (CEFIPRA) for funding this work.

\section{REFERENCES}

[1] D. M. Boyd and N. B. Ellison. Social network sites: Definition, history, and scholarship. Computer-Mediated Communication, 13:210-230, Oct. 2007.

[2] D. L. Hansen, B. Shneiderman, and M. A. Smith. Social Media: New Technologies of Collaboration in Analyzing Social Media Networks with NodeXL: Insight from a Connected World. Morgan Kaufmann, Boston, 2011.

[3] N. B. Ellison, C. Steinfield, and C. Lampe. The benefits of facebook friends: Social capital and college students use of online social network sites. Computer-Mediated Communication, 12:1143-1168, July 2007.

[4] Jakob Nielsen. Scrolling and attention. 2010. Available at http://www.nngroup.com/articles/scrolling-and-attention/.

[5] Eitan Altman. A stochastic game approach for competition over popularity in social networks. Sep. 2012. Available at http://hal.inria.fr/hal00681959.

[6] Eitan Altman. A semi-dynamic model for competition over popularity and over advertisement space in social networks. In VALUETOOLS, pages 273-279, 2012

[7] Opher Baron, Oded Berman, and David Perry. Shelf space management when demand depends on the inventory level. volume 20, pages 714-726. Wiley Online Library, 2011.

[8] Eitan Altman and Alex Reiffers. A mean field approach for the analysis of a timeline game. 2013. In preparation.

[9] S. Buck. The beginners guide to facebook, May 16, 2012 [April 24, 2013]. 